\title{
Visualization of the Effect of Topography on Connected Vehicle Communications using LiDAR-derived Models and Interactive Mapping Techniques
}

\author{
Leslie C. Harwood $^{1 *}$ and Zachary R. Doerzaph ${ }^{2}$ \\ ${ }^{1,2}$ Virginia Tech Transportation Institute \\ Ilharwood@vtti.vt.edu, ${ }^{2}$ zdoerzaph@vtti.vt.edu
}

\begin{abstract}
The topography surrounding a connected vehicle $(\mathrm{CV})$, including roadway feature obstructions, the built environment, and vegetation such as trees, can negatively affect GPS receiver performance and thus the algorithms used to provide $C V$ system alerts. Therefore, it is important to understand the performance of CV systems with regard to the physical topography of the earth. Visualization of an area from the point of view of a car traveling along a roadway can be useful in communicating the role topography can play in the obstruction of $C V$ communications. In this project, a combination of tools was used to construct a LiDAR-derived raster image of a test area and derive the skyline for various points representing vehicles traveling on a roadway. Both 2-D and 3-D visualization techniques were used to develop interactive maps as an end-product. These maps allowed for visual exploration of the topography of a region alongside the data quantifying the topographical environment and $C V$ performance variables, for any point along a roadway. These visualization products allow those involved in the development of CV technology to better understand the effects that topographical obstructions can have on these systems and assist them in refining these technologies toward eventual deployment.
\end{abstract}

Keywords: airborne radar, laser radar, geographic information systems, vehicle safety, intelligent vehicles, intelligent transportation systems, connected vehicles, GPS, web mapping, data visualization

\section{Introduction}

Connected vehicle (CV) technology uses a global positioning system (GPS)-based algorithm to communicate vehicle position information wirelessly via dedicated shortrange communication (DSRC) radio or other technologies between vehicles (vehicle-tovehicle; V2V), and/or the infrastructure (vehicle-to-infrastructure; V2I), and/or other transportation system users (e.g., pedestrians, bicyclists, trains). It has been found that the topography surrounding a $\mathrm{CV}$, including roadway feature obstructions (e.g., underpasses and tunnels), the built environment, and vegetation such as trees, can negatively affect GPS receiver performance and thus the algorithms used to provide $\mathrm{CV}$ system alerts through the obstruction of a full open-sky view. Therefore, it is important to understand the performance of CV systems as they relate to the physical topography of the earth.

Visualization of an area from the point of view of a car traveling along a roadway can be useful in communicating the role topography can play in the obstruction of CV communications. In this project, both two-dimensional (2-D) and three-dimensional (3D) visualization techniques were explored to represent the earth's topography and derived information on viewable sky area for points along a roadway. Using a combination of tools, a LiDAR-derived raster image of a test area was constructed, allowing for elevation

Received (July 28, 2017), Review Result (September 21, 2017), Accepted (October 12, 2017)

${ }^{*}$ Corresponding Author 
measurements of roadway feature obstructions, buildings, and surrounding foliage. This raster was then used to determine the point at which the topographical surface was separated from the sky (i.e., the last point of occlusion) surrounding points along a roadway. This method essentially traced the skyline of the area surrounding each point on the roadway and provided quantifiable data with regard to sky occlusion, including the percentage of open sky and mean zenith angle. Both 2-D and 3-D mapping were considered and developed for visualizing the results. In the 2-D representation, an interactive web map was developed. The 2-D web map allowed users to view the traced skyline and polar graphs for each point, along with the resulting variables. A 3-D web map was also developed in ESRI City Engine. The 3-D web map included extruded buildings and other transformed features, such as trees and roadways. This map allowed users to explore how these obstructions affected GPS signal from the point of view of a vehicle on a roadway in an immersive 3-D environment.

These methods of visualization allow a user to visually explore the topography of a region alongside the data quantifying the environment and $\mathrm{CV}$ performance variables, from the perspective of a CV. These visualizations allow those involved in the development of $\mathrm{CV}$ technology, including researchers and automotive manufacturers, to better understand the effects that topographical obstructions can have on these systems. The end-products of this project were ultimately used by $\mathrm{CV}$ technology developers to identify specific types of environments where further testing of $\mathrm{CV}$ systems was needed (e.g., areas of high occlusion from buildings, such as deep urban environments). This testing was performed under a subsequent project such that developers of $\mathrm{CV}$ technologies could refine the systems toward eventual deployment.

The work described herein was part of a larger research project that sought to correlate $\mathrm{CV}$ alerts with surrounding topography [1]. It is important to note that while current CV systems in the U.S. and Europe utilize multiple satellite-based navigation systems, including GLONASS and Galileo, during the data collection portion of the research project, GPS was the only satellite-based navigation system used to provide location information for the vehicles in this study. The detailed background and methods used to prepare the layers of geographic information described in this paper are described in more depth in the Proceedings of the 2017 Annual ESRI User Conference [2].

The following provides a review of relevant literature and methods used to create the topographical model and develop 2-D and 3-D web maps to display the outputs of the overall research project.

\section{Review of Relevant Literature and Map Development Platforms}

This project required a highly-accurate topographical model of the environment in which the CV alerts were collected to be developed. Various methods have been explored to model the surface of the earth, including types of data acquisition methods and resulting products. This project also sought to determine the best display of the topography surrounding CVs along with vehicle- and time-specific performance variables, such as vehicle speed and acceleration (lateral and longitudinal). Therefore, literature regarding the datasets available for modeling the earth's topography as well as the display of spatial data and geospatial mapping products were evaluated to help refine an end product for viewing and interacting with the results of this project.

\subsection{Datasets for Modeling the Environment}

A digital surface model (DSM) represents the earth's surface including all objects which lie on it (including buildings and vegetation). This is distinguished from a digital elevation model (DEM) or digital terrain model (DTM) which may only represent the height of the earth's surface (bare earth) (Figure 1). 


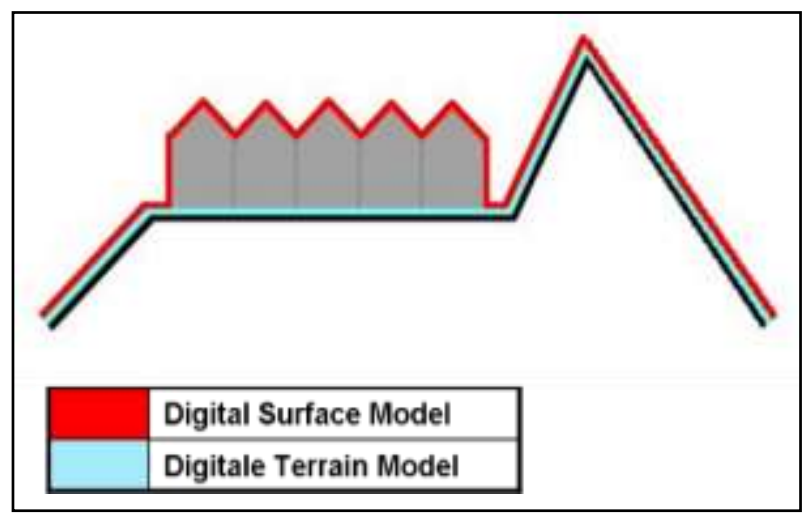

\section{Figure 1. Surfaces Represented by a Digital Surface Model (DSM) and Digital Terrain Model (DTM) [3]}

A study published in 2005 and 2007 [4,5] discusses two methods of modeling topography as DSMs: digital photogrammetry and LiDAR. Photogrammetry uses overlapping photographs to create lines of sight which are mathematically intersected to produce a 3D image and employs triangulation to precisely estimate positioning information. LiDAR is a remote sensing method of collecting highly accurate distance measurements using light and is often used to make high-resolution elevation maps. The LiDAR data discussed in this study is aerially-collected, or airborne laser scanning (ALS) data. ALS refers to data which is collected by emitting a laser pulse from a plane-mounted sensor and measuring the reflection of that pulse to the ground (bare-earth) as well as the earth's topographical surface (tops of trees, buildings). LiDAR can also "see" through vegetation, returning multiple pulses as it reflects off the surfaces encountered along its path before hitting the ground (e.g., leaves and branches). This study determined that while both photogrammetry and LiDARderived DSMs can offer a very high accuracy of modeling an environment, LiDAR is more productive due to having less time and effort involved in acquiring terrain data, especially for large areas. This study was focused on modeling terrain for predicting GPS satellite availability and ultimately determined that LiDAR was the best dataset for modeling terrain for this purpose.

$\mathrm{Li}, \mathrm{Zhu}$, and Gold [6] discuss data acquisition techniques with respect to digital terrain modeling (DTM). While DTMs differ from the DSMs of interest in this study, further investigation was performed to determine the feasibility of each of the reviewed data acquisition methods for the creation of a high-resolution (cm-level) DSM suitable for a large area. Table 1 is derived from this study with the inclusion of suitability for high-resolution DSMs covering a large application domain. The various methods reviewed include Interferometric Synthetic Aperture Radar (InSAR) and radargrammetry. The table indicates that while InSAR and radargrammetry both could be good candidates for creating a DSM, the accuracy of these methods is not high enough to create a DSM of the high-resolution necessary for this research. Conversely, some of the methods with the highest accuracy in the table, traditional and GPS surveying, have slow acquisition speeds and are only appropriate for a small area of interest. The assessments depicted in bold within this table represent the best rankings within each category, ultimately indicating LiDAR or photogrammetry as the best candidates for the creation of a high-resolution DSM for a large area of interest when cost is ignored as a factor. In addition, a study by Gamba and Houshmand [7] comparing interferometric synthetic aperture radar (InSAR or IFSAR) and LiDAR data for use in creating DSMs showed that LiDAR data provide better shape characterization of individual buildings, not only because of the data's higher 
resolution but also because InSAR data's shadowing/layover effects can only be partially corrected by segmentation procedures.

During the course of this project, it was determined that LiDAR was available for the area of interest of this study and could be used to create a high-resolution DSM.

Table 1. Comparison of DSM Data Acquisition Methods (derived from [6])

\begin{tabular}{|c|c|c|c|c|c|}
\hline Acquisition Method & Accuracy of Data & Speed & Cost & $\begin{array}{c}\text { Application } \\
\text { Domain }\end{array}$ & $\begin{array}{c}\text { Suitable for } \\
\text { high- } \\
\text { resolution } \\
\text { DSM over a } \\
\text { large area } \\
\end{array}$ \\
\hline LiDAR & High (cm) & Fast & High & $\begin{array}{l}\text { Medium to } \\
\text { large areas }\end{array}$ & Yes \\
\hline Traditional Surveying & High (cm-m) & Very slow & Very high & Small areas & No \\
\hline GPS Survey & Relatively high (cm-m) & Slow & Relatively high & Small areas & No \\
\hline Photogrammetry & Medium to high (cm-m) & Fast & Relatively low & $\begin{array}{l}\text { Medium to } \\
\text { large areas }\end{array}$ & Yes \\
\hline Space Photogrammetry & Low to medium (m) & Very fast & Low & Large areas & No \\
\hline InSAR & Low (m) & Very fast & Low & Large areas & No \\
\hline Map Digitization & Relatively low (m) & Slow & High & Any area size & No \\
\hline Map scanning & Relatively low (m) & Fast & Low & Any area size & No \\
\hline Radargrammetry & Very low $(10 \mathrm{~m})$ & Very fast & Low & Large areas & No \\
\hline
\end{tabular}

\subsection{Display of Spatial Data}

The work described herein ultimately focused on developing a geospatial mapping product through which the results of the analyses conducted may be effectively communicated with researchers and the sponsor. The development of this visualization tool was aimed at allowing future researchers and manufacturers of CV technologies to easily view and understand the results of the overall research project. While impossible to delve into the vast literature regarding cartography, and for simplicity, let it be understood that maps can be conveyed in one of two media forms; print, or physical (e.g., a globe), and digital. The data resulting from this research regarding CV alerts and topographical obstructions is complex and not easily conveyed by conventional print or physical means, and thus must be displayed digitally in order to communicate this information effectively.

In 2006, an article in Nature touted the rapid growth in the use of digital globe software, namely Google Earth, and its emerging application for researchers in all fields of study [8]. The article quotes David Whiteman, an atmospheric scientist at NASA's Goddard Space Flight Center, on the use of this digital mapping technology, emphasizing that three-dimensional visualization of data is "about more than just creating images", but also about "getting an intuitive grasp of the problem, the measurements, and the analytical challenge" [8]. Google Earth and similar globe-like digital visualizations of spatial data are a common media among both researchers and laymen. While physical maps are still required in some forms of information dissemination (e.g., journals or books), the ease of use of this type of software to share, display, and understand spatial data makes digital map forms the clear choice, when a physical map is not required. In addition, a sub-type of digital mapping media, web mapping, is currently flourishing. The following subsection briefly describes web mapping techniques and recommends those applicable to achieve the goals of the proposed research project.

2.2.1. Web Mapping: Neumann [9] describes web mapping as "the process of designing, implementing, generating, and delivering maps on the World Wide Web". 
Internet-based maps are one of the most efficient ways of disseminating mapping data and [9] notes that this medium allows for many new opportunities, such as:

- real-time maps,

- lower costs involved in map dissemination,

- less-costly and more frequent updates of data and software,

- personalized content,

- distributed data sources, and

- sharing of geographic information (spatial data).

Along with these opportunities, Neumann notes several challenges, including:

- technical limitations such as low resolution and limited bandwidth,

- issues with copyright and security,

- issues regarding reliability, and,

- technical complexity.

The author categorizes eleven types of web maps and provides a figure ranking the properties of web maps based on increasing sophistication. A 2-D or 3-D interactive, web-based map was envisioned to meet the goals of this research. Neumann describes interactive web maps as allowing users to explore, change parameters, and navigate/interact with the map, as well as possibly reveal and hide additional information and link to other resources, etc. The technical back-end required to implement such a type of map is achieved through events, scripting, and Document Object Model (DOM) manipulation, support for which is included in all modern web browsers [9]. The author explains that DOM combined with scripting is known as Dynamic HTML (DHTML). DHTML is a commonly-used language that Google Maps and other well-known mapping sites use in combination with other languages and network requests to create interactive maps on their websites.

The recognized GIS industry-leader, ESRI, has developed a software package which allows users to build and publish 3-D web maps directly to the web with relative ease. CityEngine also uses HTML and can be shared privately via a viewer application which can be uploaded on web servers of private organizations [10]. CityEngine also has a pointed integration with other ESRI ArcGIS products and welldocumented methods to facilitate transforming, updating, and publishing map layers through the use of these two ESRI products. It was determined that ESRI ArcGIS software would be used for development of mapping layers through the spatial analysis performed under this project and that ESRI CityEngine be used to develop the 3-D interactive, web-based map.

\subsection{Summary}

The literature reviewed concluded that the topography within the area of interest of this project could be effectively modeled via a DSM using LiDAR data. This review also provided a discussion of mapping methods and software able to meet the goals of this research, with the determination of suitable methods for developing web maps for this project. 


\section{Data Acquisition}

During this study, CV alert location and performance variables were collected [2]. In addition, LiDAR data were obtained for use in subsequent analyses. A description of the data acquired for use in this project is included below.

\subsection{Alert Location and Performance Variables}

During the larger research project described in [2], CVs were instrumented with data acquisition systems (DAS) and communicated with other vehicles and the infrastructure (V2V and V2I) during a year-long data collection period. The DAS on these vehicles captured corresponding location and performance variables where CV safety application alerts were provided to drivers during this period. The location variables from this dataset which were used in the analysis portion of this project were the GPS latitude and longitude of each vehicle; however, other location variables were used in the visualization of the final results, including:

- Horizontal Dilution of Precision (HDOP)

- Position Dilution of Precision (PDOP)

- GPS Coasting (error measure as a result of antenna or receiver failure, or if an antenna is fully blocked from view of any satellite)

- Fix Quality

- Number of Visible Satellites

Performance variables were also collected from each CV via the DAS. While these variables were not directly used in the analyses described herein, they were used in the web map creation portion of this study, allowing for visualization of the effects of topography on CV communications. These variables included:

- Vehicle-related information, such as:

○ Speed

- Lateral Acceleration

- Longitudinal Acceleration

○ Throttle Position

- CV-related information, such as:

- Alert validity (valid, invalid)

○ Causations of invalid alerts (algorithmic, environmental, inconclusive)

\subsection{LiDAR Data Acquisition}

For this analysis, publically-available aerially-collected LiDAR data were obtained. An approximate $15.5 \mathrm{~km}$ by $14 \mathrm{~km}$ cordon was created around the geographic area of interest. A total of 120 tiles of LiDAR point cloud data covering the area of interest were collected in LAS file format (the public, industry-standard binary file format for storing aerially-collected LiDAR point cloud data [11]. The LiDAR data used in this project were collected in 2009 through a United States Geological Survey (USGS) contract. The contractor used a LH Systems AL850 LiDAR system, collecting multiple returns for each laser pulse as well as an intensity value for each return over an 18-day period from April 16, 2009 to May 3, 2009. Data 
were acquired at a height of 7,000 feet above ground level at a flight speed of 120 knots, with a scanner field of view of 14 degrees and a swath width of 1,719 feet. The data collected under this contract was in accordance with National Standard for Spatial Database Accuracy standards and provides precise spatial information about the shape and surface characteristics of this area. The horizontal accuracy of this data is \pm 3.8 feet at the 95th percent confidence level with a Root Mean Square Error (RMSE) of 1.7 feet and it tested 0.56 feet vertical accuracy at the 95th percent confidence level for all land cover categories with a RMSE of 0.29 feet. Intensity information captured by the LiDAR system is related to the reflectivity of a surface; intensity indicates the relative energy returned to the sensor from the reflective surface pulse. It is important to note that the intensity information captured was not calibrated or normalized; however, a calculated correction value (auto gain control) is applied to each intensity value in order to continually center the intensity values on an intensity histogram [12]. A derivation of this data set was then used to model the topography within the area of interest as described in Section 4.

\subsection{Data Acquisition for Web Map Development}

Additional data acquisition was required to complete the 3-D web map, as desired for this research project's end-product. In addition to the LiDAR-derived DSM described in Section 4, 1/9 arc-second USGS digital elevation models (DEMs) were collected from The National Map Viewer. These DEMs provided approximately 3 meter-level resolution elevation data. In addition, shapefiles representing building footprints and city-owned trees were obtained from local governments within the area of interest. After LiDAR acquisition was completed within the area of interest in 2009, the contractor used the LiDAR data and existing 2005-2008 orthoimagery to create building footprints using a semi-automated method [12]. Finally, TIGER street line files covering the area of interest were downloaded from the U.S. Census Bureau website.

\section{Methods and Results}

This project required that the topography in the area of interest be modeled so that the environment surrounding $\mathrm{CV}$ alert locations could be assessed. Through the literature review, it was determined that aerially-collected LiDAR could be used to model a topographical environment. This model (raster) was then used in conjunction with CV alert locations (points) to quantify the environments in which CV alerts occurred. The variables describing the topographical environment as well as other layers of spatial data were then symbolized and prepared for visual representation and development of interactive maps. The methods used to create the model of the topographical environment, quantify the environment, and prepare data and create interactive 2-D and 3-D maps are described in the following sections.

\subsection{LiDAR-derived Raster Development and 3-D Analysis to Quantify Topography}

The natural topography within the area of interest was relatively flat, with a ground elevation gain of only approximately 110 meters (361 feet). However, ground elevation does not account for roadway features, buildings, trees, and other obstructions which may also diminish GPS accuracy by impeding the reception of signals from the full constellation of available satellites. As determined in the literature review, LiDAR data form a high-accuracy, high collection-speed dataset for use in modeling the earth's topography, including the terrain, roadway feature obstructions, built structures, and vegetation, over a large area of interest. 
The LiDAR data were acquired for this project in LAS format. Using ESRI ArcGIS's LAS Dataset to Raster toolset, the first-return points in the data were used to create a raster DSM representing the topography in the area of interest. This was accomplished by running the tool using nearest neighbor cell assignment and natural neighbor interpolation for void fills, and a one-foot cell size sampling value to define the raster resolution. The resulting DSM is depicted in Figure 2.

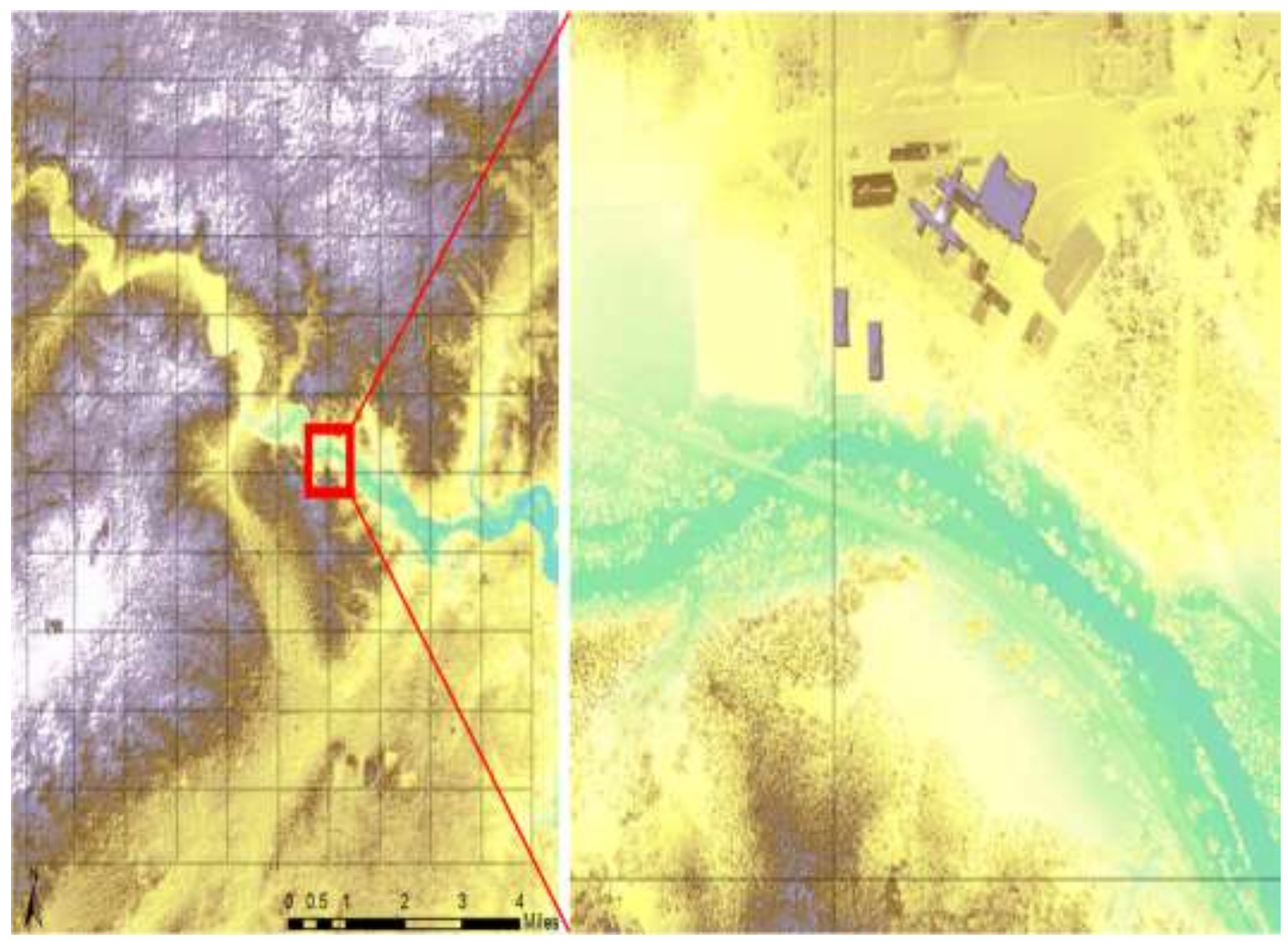

\section{Figure 2. A One-Foot Resolution Raster DSM using First Returns Derived from the LAS Dataset}

The CV alert locations (latitude and longitude) were then overlaid on the resulting DSM for further analysis using ESRI ArcGIS software. In order to quantify the topographical environment, the authors used the ESRI Visibility toolset in the 3-D Analyst extension. Using the Skyline and Skyline Graph tools, the skyline of the area in the DSM surrounding each point was defined ("looking" 360 degrees horizontally around each point in one-degree increments and at all vertical angles to determine the point at which the topographical surface is separated from the sky [last point of occlusion]) as depicted in Figure 3. The results from this analysis were then summarized to obtain the following variables about the topographical environment surrounding the alerts:

- Percent Open Sky ("viewable" portion of the sky with a vertical baseline angle of zero $(0)$

- Percent Shade (the inverse of the Percent Open Sky)

- Maximum Zenith Angle (the maximum angle to an occlusion in the skyline of an alert point)

- Mean Zenith Angle (the mean angle to occlusions in the skyline of an alert point) 


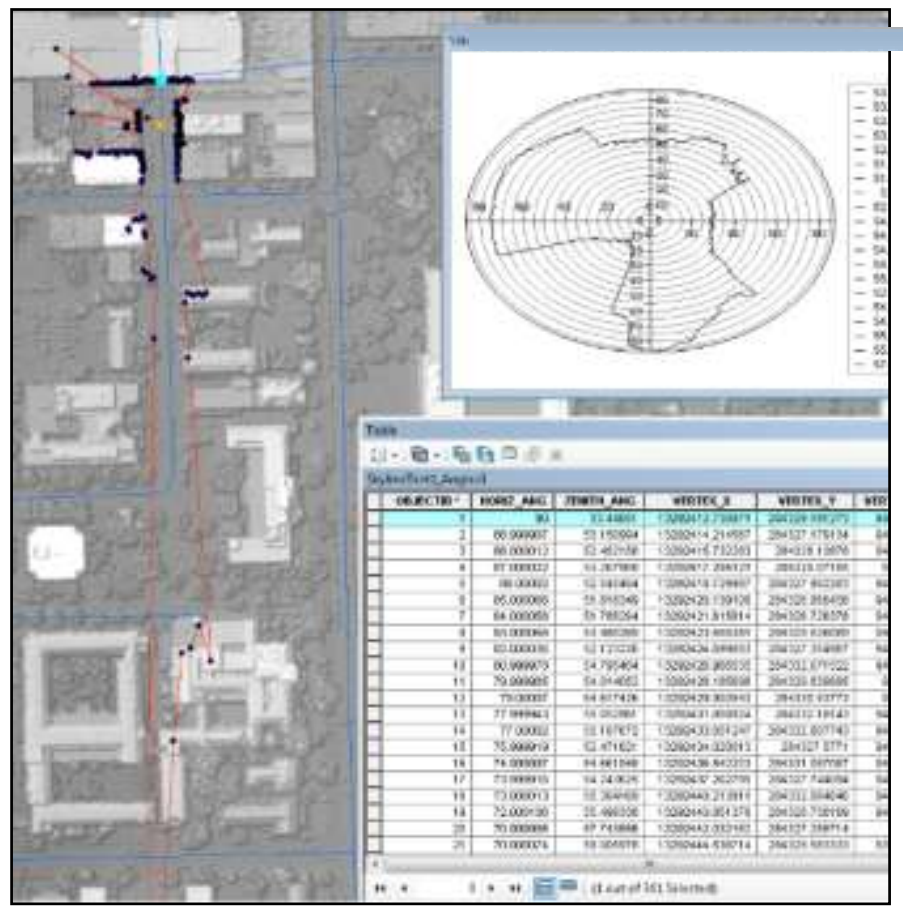

Figure 3. A Screenshot of the Results from the ArcGIS 3D Analyst Extension "Skyline" and "Skyline Graph" Tools (Orange Dot on Roadway Representing an Alert Point [top, left], Surrounding Points in Dark Blue Representing the Vertical Point of Occlusion at One-Degree Horizontal Angle Increments [left], the Table Representing the Tabular Results for each Point [bottom, right], and a Polar Graph Representing the Viewable Sky for a Given Point [top, right])

Harwood \& Doerzaph [2] describes in detail the spatial analysis performed on this raster to obtain variables displayed in the final web map product of this project.

\subsection{Web Map Development and End Products}

The layers of spatial data developed using the methods described in the previous section were symbolized and prepared for 3-D web map publication using City Engine to create a proof-of-concept web map. Although multiple application programming interfaces (APIs) were considered, the final web map prototype was developed using ESRI ArcGIS and a 3-D map was published using the City Engine Web Viewer, as the literature deemed appropriate. The base map layers for this prototype web map was developed using USGS DEMs (described in Section 3.3) and a DSM derived from first-return USGS-collected LiDAR data, publically available (TIGER) roadway line files, and publically available aerial imagery.

4.2.1. 2-D Web Map: While this project focused primarily on a 3-D web map as the final product of this research, a 2-D interactive web map was also created. This web map was hosted online using ArcGIS Online. This map included point and line file IDs (FID), vehicle heights on the earth as measured in feet (OFFSETA), and the percentage of viewable sky (Skyview). To develop this map, the ArcGIS API for JavaScript was used to call the hosted map from ArcGIS Online via a map ID. This web map was built using the following layers:

- CV Alert Locations (points)

- Skyline (lines) 
Users of the 2-D web map had the ability to click on any CV alert location point to view information related to the $\mathrm{CV}$ alert given as well as the skyline trace. These layers were overlaid on aerial imagery of the area of interest, allowing viewers to see the topographical obstructions from a top-down vantage point. A screen capture of the 2-D interactive web is depicted in Figure 4. Additional information could be added to this map, allowing users to view any information of interest related to the CV alert or the surrounding topographical environment in a window within the web map by simply clicking on a point.

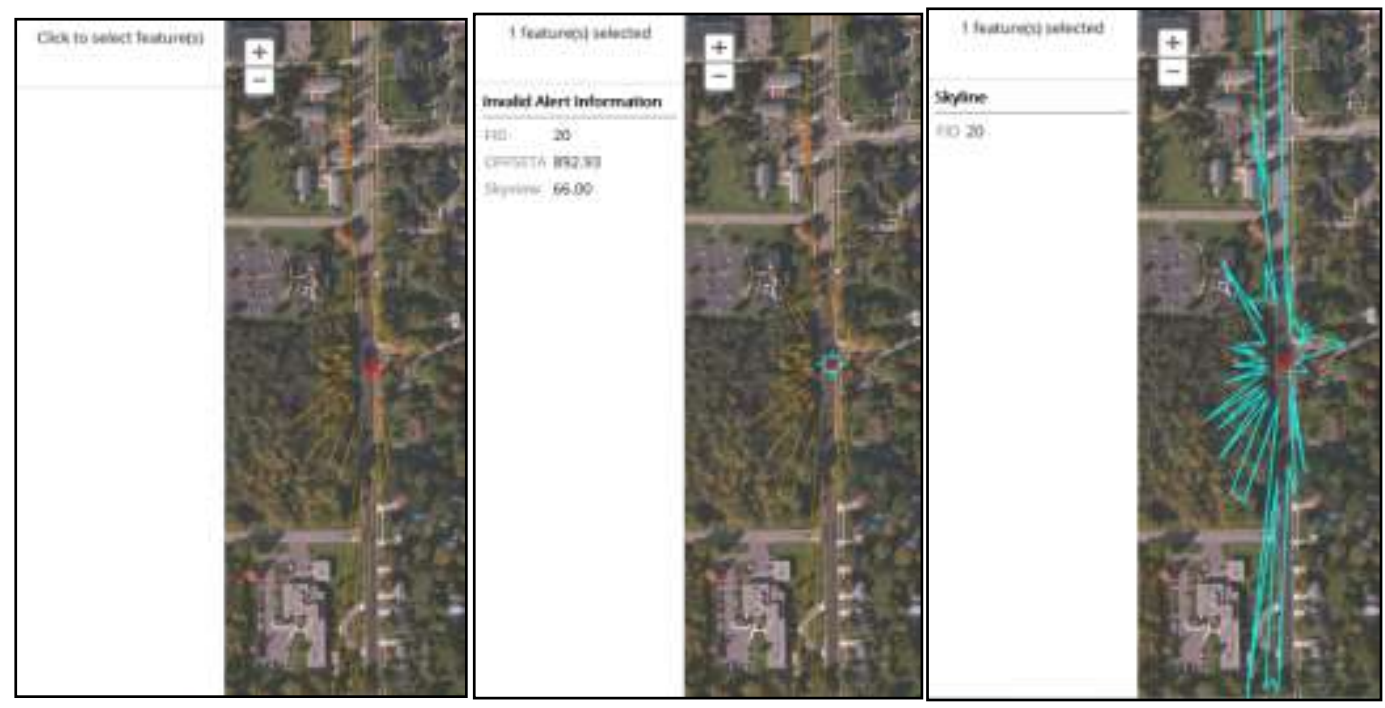

Figure 4. 2-D interactive Web Map as Viewed in a Web Browser

4.2.2. 3-D Map: A 2-D web map such as that depicted in Figure 4 is a simple interface for a novice user and not very resource-heavy to create and display this information. However, it was believed that a 3-D web map would provide a much better visualization of this data and the topographical obstructions surrounding a CV alert.

The data collected specifically for web map development under this project is described in Section 3.3. This data was symbolized and prepared for 3-D web map publication using City Engine. ESRI has completed multiple tutorials regarding data preparation, maintenance, publication, etc., for City Engine. The 3DCity Data Preparation models/tools and PDF instructions provided the primary process for preparing the data for publication

\section{Creating the 3-D Map}

The ESRI Spatial Analyst extension was used to obtain zonal statistics of raster data, including minimum, maximum, and mean elevations, for the building footprints. Zonal statistics for building footprints were obtained on the three-meter DEM for the area of interest to obtain the average building base heights and on the LiDAR-derived DSM to obtain the average building heights. The ESRI 3-D Analyst extension was then used to generate a height-, or z-, aware building footprints layer based on the average elevation for each building obtained from the DEM in the previous process. This layer was then visualized in ESRI ArcScene and extruded to the average building height obtained from the LiDAR-derived DSM. Finally, a multi-patch feature class which is an object that stores features as a collection of patches representing the boundary of a 3-D object as a single row in a database was created that represented the 3-D polygon for each building.

TIGER Streets and City trees shapefiles (2-D, as originally acquired) were then transformed into 3-D features based on the underlying USGS DEM elevation values. 
The CV alert points used were previously made z-aware through the analysis deriving skyline. The base heights of these points represented the average height of GPS antennae mounted on a CV. In addition, the lines representing the skyline, as obtained from the same skyline analysis, were also $\mathrm{z}$-aware. Therefore, no additional tools were necessary to prepare these data.

\section{Map Symbolization}

The following layers were symbolized in ESRI ArcScene:

- CV Alert Locations (points)

- Skyline (lines)

- USGS DEM (raster [TIF])
- City of Ann Arbor Trees (points)

- Streets (lines)

- Buildings (multi-patch)

Each vector layer was symbolized using 3-D symbology for compatibility with the final export. Buildings were symbolized with 25 percent transparency to enhance the visibility of the $\mathrm{CV}$ alert points and corresponding skyline polylines along roadways. The raster image was floated on itself to provide a 3-D basemap and was symbolized using a color scale complementary to the remaining layers. The result of this symbolization is shown in Figure 5.

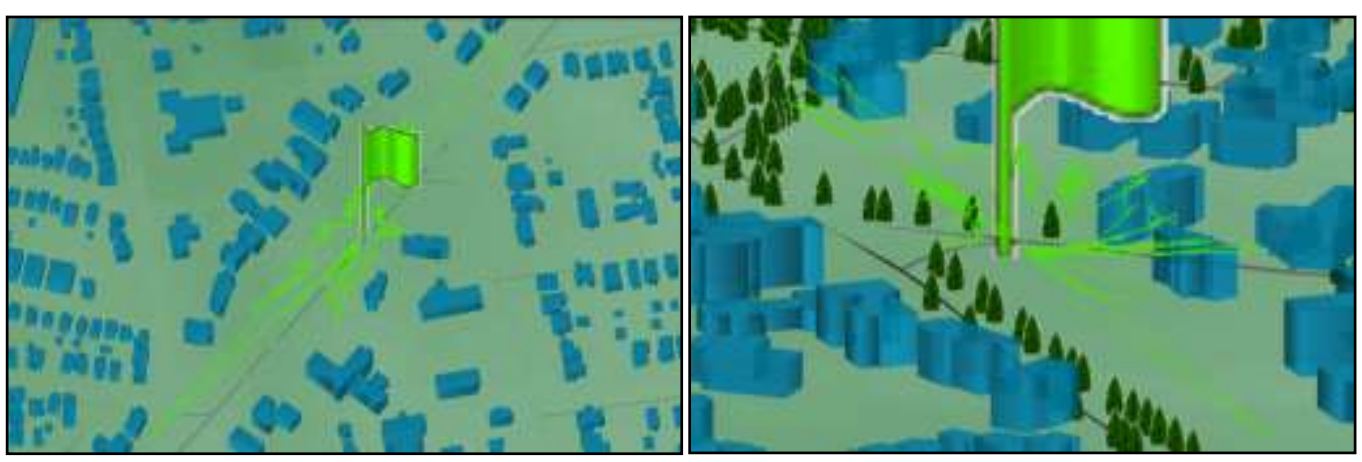

Figure 5. 3-D Symbolization of Map Features in Preparation for Web Map Development

It is important to note that, due to the spatial alignment offset of the LiDAR-derived DSM used for skyline analysis and the building footprints shapefile, the lines representing the skyline often seem to penetrate buildings when viewed in 3-D. The skyline also seems to "hit" obstructions that are not visible in the 3-D view; this is suspected to be caused by discrepancies between the DEM and LiDAR data and/or shapefiles representing buildings and trees. Buildings may have been excluded during the generation of the building footprints shapefile, and the trees shapefile is known to only depict primarily city-owned trees.

\section{Final Web Map Development}

City Engine 2013 was downloaded and installed on a local machine for use in web map development. In addition, an offline viewer package was installed to allow the display of the prototype web map without fully publishing online. During the creation of a 3-D web scene from the symbolized layers, many issues were encountered, and only a partial web scene was able to be "published." The issues encountered included the system running out of memory while displaying layers and exporting to a 3-D web scene. One possible solution for future determination is to use a more powerful machine with more memory when performing these tasks. Unfortunately, the resources necessary to complete this task as desired were unavailable during the course of this project. 
Although a complete 3-D web scene could not be produced, the resulting partial scene provides a general understanding of the future final product. The partial 3-D web scene in Figure 6 depicts buildings (in blue as semi-transparent features) and the $\mathrm{CV}$ alert points (red and green pyramids) floated on top of the symbolized DEM. Each feature within the scene is interactive, with information viewable in the right-hand pane. While unable to be completed during the course of this project, it is envisioned that this web scene will eventually include 3-D roads and skylines for each safety alert, as well as other pertinent information.

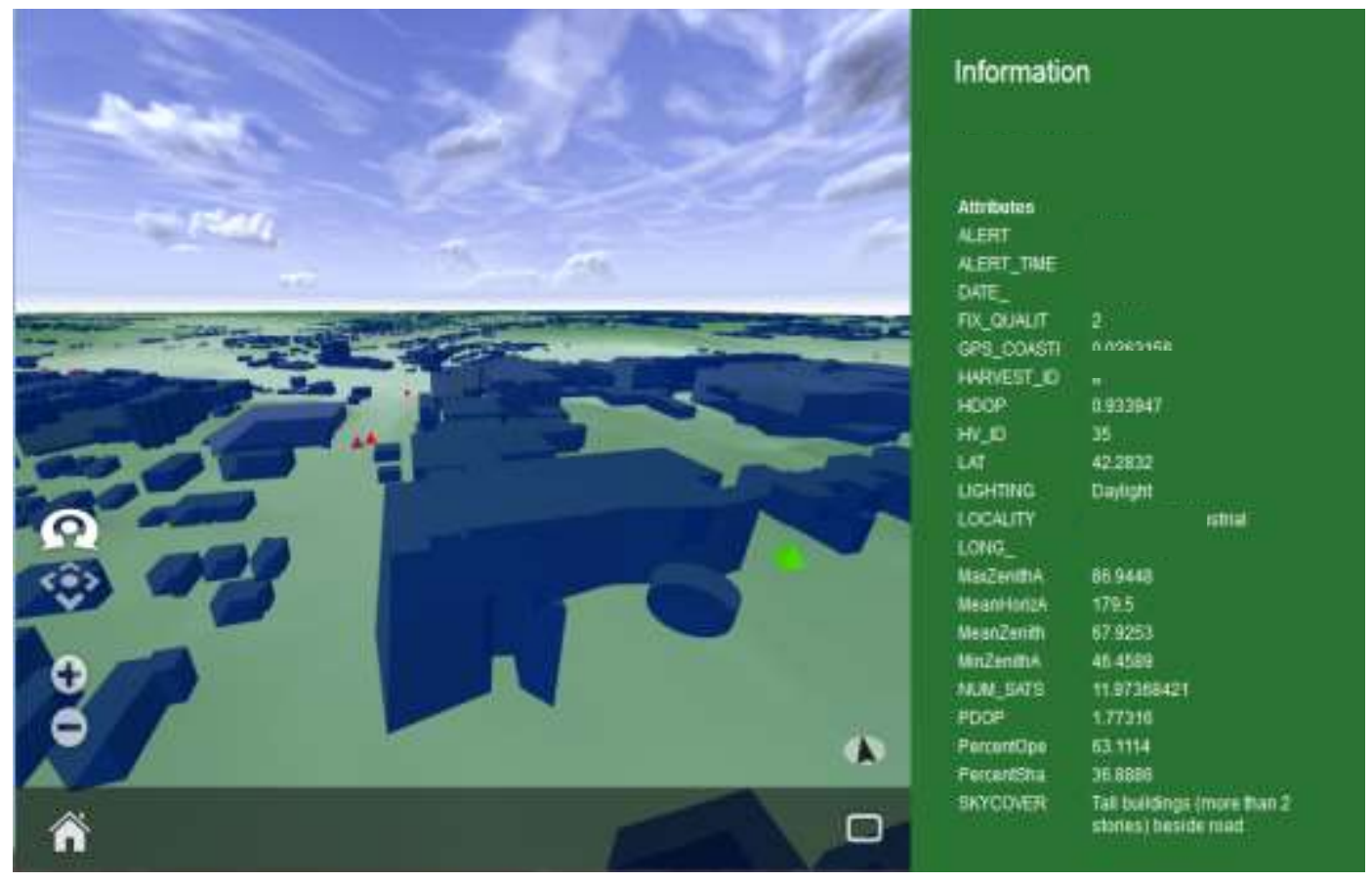

Figure 6. Partial CityEngine 3-D Web Scene as viewed in the Offline Viewer

The resulting 3-D web map is a more advanced exploration than the 2-D web map and is familiar to most users (i.e., is navigable in a similar way to Google Earth, a commonly used product). However, the methods used to create this 3-D map for this project were resource-intensive and the authors encountered issues due to processing power and available memory.

\section{Discussion}

This project was focused on using LiDAR-derived models and other geospatial data products to model the topography surrounding CVs traveling along a roadway and creating web mapping products to explore ways to communicate the effects that topography has on CV communications with $\mathrm{CV}$ technology developers.

The topography of the area of interest was successfully modeled using publically available LiDAR datasets and two web-mapping products were ultimately created to explore ways to effectively communicate geospatial and statistical analysis results with researchers. A 2-D interactive web map was created, which included exploring the use of the ArcGIS API for JavaScript for development of a web-based interface.

In addition, a 3-D web map was prepared. Although ultimately only a partial 3-D scene could be created during this project, the result provided a general understanding of the future final product and proved that the method of using a CityEngine Web Scene published on a web server and displayed through a Viewer application has great potential for effectively communicated the overall research project results. The 
problems experienced during the creation of the 3-D web scene were largely due to system processing power and memory limitations.

While many advantages exist for using a 3-D versus a 2-D web map to display the results of this project, the 2-D product may serve as a suitable alternative in the event that a 3-D web map ultimately cannot be created.

\section{Limitations of Approach and Future Work}

The topographical model was developed using a publically-available LiDAR dataset. LiDAR is often acquired during a short period (e.g., one month). This finite period of data collection can present issues regarding matching CV data with LiDARderived topographical models due to environmental changes, such as variations in vegetation (i.e., leaf-on vs. leaf-off periods) and changes in the built environment (e.g., new structures built or removed during the data collection period). An ideal aerially-collected LiDAR dataset would thus correspond to the exact data collection period. Future analyses will focus on matching the CV data collected with the time period that the aerially-collected LiDAR was/is available.

As stated, the authors were unable to achieve complete results for the 3-D mapping product. This partial result provided a general understanding of what the ultimate 3$\mathrm{D}$ product would convey. It is believed that using a more powerful machine (i.e., a machine with more processing power and available memory) when performing the tasks required to create the 3-D web map should eliminate these issues. However, other products have since been released which should be explored for creating the 3D web map, including ArcGIS Pro. The authors expect that the improved visualization products which have been released since the time that this project was conducted will allow for a complete 3-D mapping product.

In addition, $\mathrm{CV}$ technologies have improved since the time this project was conducted. Environments where CVs had issues were identified using the work described herein, and additional testing and technology refinement has occurred to largely overcome these issues, where possible. The future work listed above will be useful in continually refining $\mathrm{CV}$ technologies. These methods will ultimately provide $\mathrm{CV}$ technology developers the ability to better assess the effects that topography has on $\mathrm{CV}$ technologies through advanced visualization techniques, with the goal of further identifying testing environments toward CV technology refinement and eventual CV deployment.

\section{Acknowledgments}

The authors would like to thank the Peter Sforza, Director of the Virginia Tech Center for Geospatial Information Technology, for guidance in developing the web mapping products associated with this work.

\section{References}

[1] L. Harwood (unpublished), "Identifying Environments in which Vehicle-to-Vehicle Safety Application Alerts were affected by the Surrounding Topography", Masters Thesis, Virginia Tech, Blacksburg, Virginia.

[2] L. Harwood and Z. Doerzaph, "Predicting Connected Vehicle Alert Classification Based on Topography", Proceedings of the 2017 Annual ESRI User Conference, San Diego, USA, (2017).

[3] M. Over, "Surfaces represented by a Digital Surface Model and Digital Terrain Model (via Wikimedia Commons)", Retrieved December 2, 2014, from http://commons.wikimedia.org/wiki/ File:DTM_DSM.png, (2011).

[4] G. Taylor, J. Li, D. Kidner and M. Ware, "Surface Modelling for GPS Satellite Visibility", In K.-J. Li \& C. Vangenot (Eds.), Web and Wireless Geographical Information Systems, Springer Berlin Heidelberg, vol. 3833, (2005), pp. 281-295. 
[5] G. Taylor, J. Li, D. Kidner, C. Brunsdon and M. Ware, "Modelling and prediction of GPS availability with digital photogrammetry and LiDAR", International Journal of Geographic Information Science, doi: 10.1080/13658810600816540, vol. 21, no. 1, (2007), pp. 1-20.

[6] Z. Li, C. Zhu and C. Gold, "Digital terrain modeling: principles and methodology", CRC press, (2010).

[7] P. Gamba and B. Houshmand, "Digital surface models and building extraction: a comparison of IFSAR and LIDAR data", Geoscience and Remote Sensing, IEEE Transactions, vol. 38, no. 4, (2000), pp. 19591968.

[8] D. Butler, "Virtual globes: The web-wide world", Nature, vol. 439, no. 7078, (2006), pp. 776-778.

[9] A. Neumann, "Web Mapping and Web Cartography", Springer Handbook of Geographic Information, Springer, (2012), pp. 273-287.

[10] M. DeMeritt, "Transform 2-D Data into 3-D City Models Faster with Esri CityEngine 2012", Retrieved February 14, 2014, from http://www.esri.com/news/releases/12-4qtr/transform-2-D-data-into-3-D-citymodels-faster-with-esri-cityengine-2012.html, (2012).

[11] ASPRS, LASer (LAS) File Format Exchange Activities. Retrieved November 1, 2014, from http://www.asprs.org/Committee-General/LASer-LAS-File-Format-Exchange-Activities.html, (2013).

[12] USGS, Metadata for MI_Wayne-WashtenawCo_2009. Retrieved 2009-08-31, from United States Geological Survey (USGS), (2009).
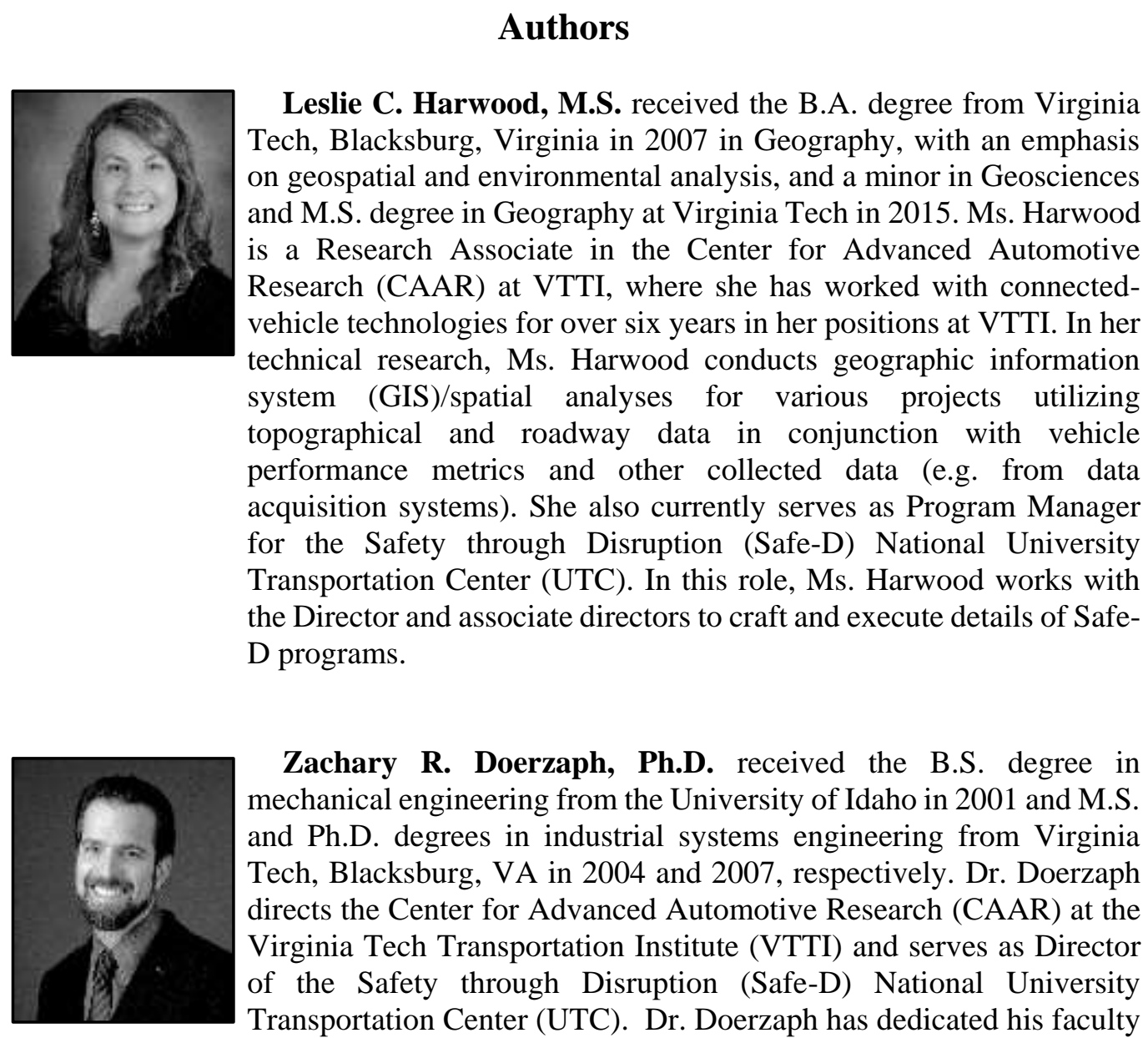

Zachary R. Doerzaph, Ph.D. received the B.S. degree in mechanical engineering from the University of Idaho in 2001 and M.S. and $\mathrm{Ph} . \mathrm{D}$. degrees in industrial systems engineering from Virginia Tech, Blacksburg, VA in 2004 and 2007, respectively. Dr. Doerzaph directs the Center for Advanced Automotive Research (CAAR) at the Virginia Tech Transportation Institute (VTTI) and serves as Director of the Safety through Disruption (Safe-D) National University Transportation Center (UTC). Dr. Doerzaph has dedicated his faculty career to advanced transportation systems in accordance with his genuine belief that technology represents the future of our transportation system. As Director of CAAR and Safe-D, he coordinates a research portfolio focused on measuring and improving the safety and performance of next generation vehicle systems, including connected vehicles, collision avoidance systems, automated driving systems, driver interfaces, and driver behavior monitoring and evaluation. 